Refusals to Approvals: Ensuring Submission Quality \title{
Santosh Tymms
}

\section{Background}

Only $50 \%$ of NME applications are approved on first submission to the FDA, with a median delay of 435 days to approval following the first unsuccessful submission. Resubmission of failed applications is costly and delays the availability of vital new drugs to patients. Twelve reasons for regulatory submission failure related to efficacy and safety have been identified via Sacks et al (2014).

For each of these reasons, Quanticate are devising a list of statistical and programmatical data checks, post database lock, to identify when potential submission failure may occur, along with reasons and explanations where possible. With a clearer understanding of why the data is the way it is, any potential findings can be explained and discussed in the CSR ahead of submission to mitigate any potential chance of failure.

To create this list, statisticians within Quanticate were interviewed about the failure reasons and asked their opinion on appropriate checks for each reason. The responses were then aggregated. The research was then extended to generate further ideas and gather expert views from statisticians and other professionals involved in clinical trial submissions outside of Quanticate. The hope was to further aggregate these responses in a Delphi style piece of research.

Reference: Leonard V. Sacks et al., MPH Scientific and Regulatory Reasons for Delay and Denial of FDA Approval of Initial Applications for New Drugs, 2000-2012 (2014) JAMA. 2014;311(4):378-384

doi:10.1001/jama.2013.282542

\section{Results}

A rich a varied set of responses were gathered within Quanticate, but Delphi research responses outside of Quanticate were lower than hoped. The following provides a sample of aggregated suggested checks by failure reason.

\section{Data Integrity and Missing Data}

Checks:

- CDISC compliance

- eCTD format compliance

- Determine anomalies and extent of and patterns in missing data, e.g. points at which compliance starts to slip, or if missing data affects one group more than others, using:

$$
\text { - SAS JMP }
$$$$
\text { CluePoints }
$$

- Investigate impact of the missing data using - Imputation

- Tipping Point Analysis

Output from CluePoints Showing Anomalous Sites and Anomalous Data at a Given Site

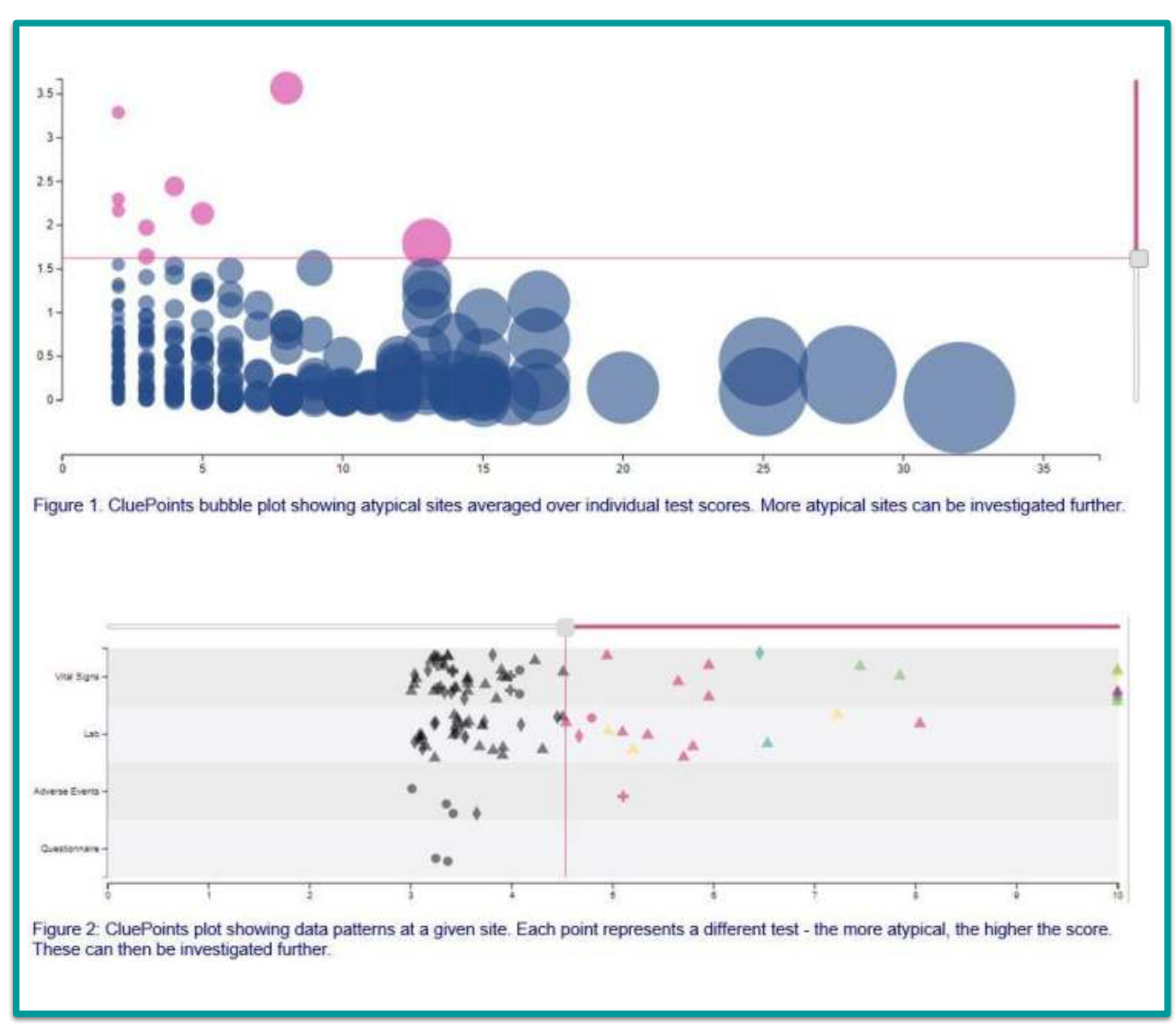

\section{Safety Deficiencies}

Checks:

- Examine distributions of AEs (all, severity, seriousness, etc.) across all sites, regions, studies and populations (predefined and post hoc)

- Have unusual Phase 2/3 patterns in AEs been investigated in Phase 1

- Check conflicting safety data in Phase 2/3 been retrospectively investigated in Phase 1 - Examine consistency of population across studies (ISS). If the population differs then pooling results might not be plausible (e.g different study pools would be required)

Output from SAS JMP Showing Spread of Adverse Events

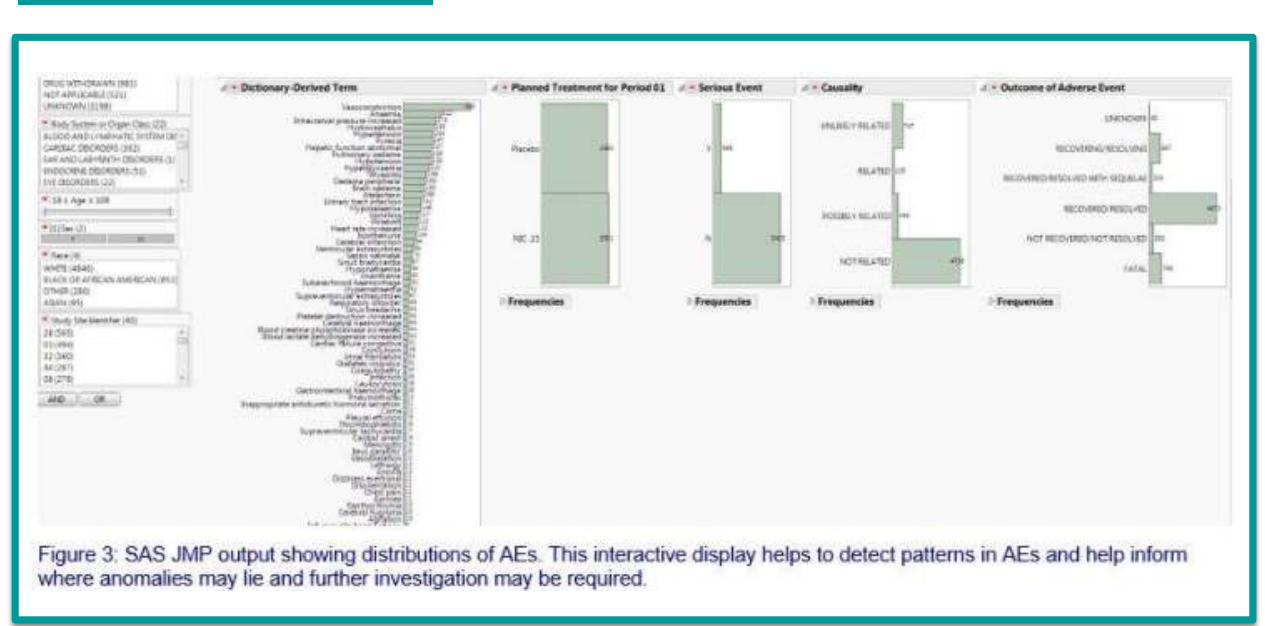

Inconsistent Results for Different Endpoints

Checks:

- Carry out efficacy analyses (primary secondary etc.) on various sub-populations

- Compare efficacy results in each study (ISE) - Were any populations identified afterwards that weren't planned for? Are new and old populations demonstrated and explained in the CSR

-Was the trial powered for secondary endpoints - Look at distribution of endpoints across studies, regions and populations

\section{Other Failure Reasons}

Other reasons include:

- Population does not appropriate to reflect intended use,

- Population too small to demonstrate efficacy

- Uncertainty/disagreement about dose

- Inability to determine noninferiority margin

- Confounding by concomitant medication

- Unsatisfactory endpoint

- Inconsistent results in different trials and/or study sites

- Inadequate efficacy compared with SOC

Some of these categories allow for examination of data and explanation, e.g. response rates fo those with and without concomitant medication can be investigated. However, there are cases when more data and revised analyses may be required, e.g. in cases when the population was not appropriate. In some cases it is not clear that further analysis can affect the results, e.g. inadequate efficacy.

\section{Conclusions}

- Bolstering submission by carrying out checks where failure can commonly occur is of great importance

- Aggregating opinions of experts is of real value.

To get more responses perhaps a larger audience could be targeted

- Every trial presents its own set of questions and investigations. This research is therefore in a constant state of flux and refinement 